(33\%) were found to have PN and they were distributed as follows: 2 patients $(8.3 \%)$ were proved to have chemotherapy induced $\mathrm{PN}$, 4 patients $(16.6 \%)$ showed subclinical chemotherapy induced $\mathrm{PN}$ and 2 patients (8.3\%) showed subclinical PN due to ALL itself. 5 cases $(62.5 \%)$ were found to have axonal pattern of PN, 1 case $(12.5 \%)$ was found to have demyelinating pattern of $\mathrm{PN}$ and 2 cases (25\%) were found to have mixed axonal demyelinating pattern of affection. Motor nerves affection was greater than sensory nerve.

Conclusion Chemotherapeutic agents used in phase I (induction of remission) proved to have a neurotoxic effects on peripheral nerves. In most of the patients, the peripheral neuropathy was subclinical.

\section{THE CLINICAL FEATURES AND OUTCOMES OF MOYAMOYA DISEASE IN A MEDICAL CENTER IN TAIWAN}

doi:10.1136/archdischild-2012-302724.1517

CF Hu, HC Fan, SJ Chen. Department of Pediatrics, Tri-Service General Hospital, Taipei, Taiwan R.O.C.

Background and Aims To describe the clinical features and outcomes of patients with moyamoya disease (MMD) who received surgical or medical treatment at a single institute in Taiwan.

Methods From August 2004 to September 2010, medical charts of patients with MMD (ICD-9 code: 437.5) from a medical institute in Taiwan were reviewed. Demographic and clinical characteristics, cerebral imaging files and follow-up information, and outcome were analyzed.

Results There were total 46 patients with MMD enrolled in this study. Male versus female ratio was 21 versus 25 (=1:1.2). Ages ranged from 1 to 84 year-old with the peak incidence in the 31-40 year age group (12 cases). The incidence of cerebral ischemic infarction was $75.0 \%(6 / 8)$ in the pediatric group, and $60.5 \%(23 / 38)$ in adult group; haemorrhagic stroke was $1.3 \%(1 / 8)$ in pediatric group and $26.3 \%(10 / 38)$ in adult group. Symptoms, included paralysis (76.1\%; 35/46), consciousness change (34.8\%; 16/46), headache (17.4\%; 8/46), numbness (17.4\%; 8/46), and seizure (17.4\%; 8/46). A regression analysis showed that the outcome of patients with $\mathrm{MMD}$ was negatively related to a modified Suzuki's score $(\mathrm{p}<0.05)$.

\begin{tabular}{|c|c|c|c|c|}
\hline Case & Sex Age & $\begin{array}{l}\text { Suzuki } \\
\text { gradee }\end{array}$ & \begin{tabular}{c|} 
Modified \\
Suruki grade+
\end{tabular} & Outcome \\
\hline 10 & F 340 & 60 & 40 & 10 \\
\hline 20 & F 640 & 30 & 30 & 20 \\
\hline 30 & $\mathrm{M} 490^{\circ}$ & 30 & 30 & 30 \\
\hline 40 & $F+30$ & 30 & 30 & 40 \\
\hline 50 & $\mathrm{M} 340$ & 30 & 30 & 40 \\
\hline 60 & $\mathrm{~F} 422^{\circ}$ & 32 & 30 & 40 \\
\hline 70 & $\mathrm{~F}+00$ & 30 & 30 & 40 \\
\hline 80 & $\mathrm{M} / 210$ & 30 & 30 & 40 \\
\hline 90 & $\mathrm{M} 360^{\circ}$ & 20 & 20 & 40 \\
\hline $10+$ & $\mathrm{M} 340$ & 20 & 20 & 50 \\
\hline & $\cdots$ & in & Savkend & -1 \\
\hline
\end{tabular}

Abstract 1517 Figure 1 Glasgow outcome scale v.s modified Suzuki's score
Abstract 1517 Table 1 Image modality and stroke type in a medical center

\begin{tabular}{ll}
\hline Image & \\
\hline MRA & $73.91 \%(34 / 46)$ \\
CTA & $30.43 \%(14 / 46)$ \\
Angiography & $21.74 \%(10 / 46)$ \\
Stroke type & \\
Ischemic type & $63.04 \%(29 / 46)$ \\
Haemorrhagic type & $23.91 \%(11 / 46)$ \\
\hline
\end{tabular}

Abstract 1517 Table 2 Surgical rate and Glasgow outcome scale

\begin{tabular}{ll}
\hline Surgical type & Percentage (N=46) \\
\hline Neovascularisation & $15.22 \%(7 / 46)$ \\
Removal of hematoma & $19.57 \%(9 / 46)$ \\
None & $65.22 \%(30 / 46)$ \\
Glasgow outcome scale & Percentage $(\mathrm{N}=40)$ \\
5 & $40 \%(16 / 40)$ \\
4 & $42.5 \%(17 / 40)$ \\
3 & $2.5 \%(3 / 40)$ \\
2 & $2.5 \%(1 / 40)$ \\
1 & $5 \%(2 / 40)$ \\
\hline
\end{tabular}

Conclusions $\mathrm{MMD}$ is commonly found in the Asian area, including Japan, Korea and Taiwan. However, the outcomes of patients with MMD are unpredictable. In this study, we found that the severity of $\mathrm{MMD}$ might be correlated with the scores of modified Suzuki's grading system. Therefore, the more the scores patients with $\mathrm{MMD}$ acquire, the higher risks of infarction will possibly occur in them.

\section{EPIDEMIOLOGICAL ASPECTS IN FEBRILE SEIZURES}

doi:10.1136/archdischild-2012-302724.1518

F Cora, M Moiceanu, E Buzoianu, V Hurduc, DA Plesca. Pediatrics, Children's Hospital 'Dr. Victor Gomoiu', Bucharest, Romania

Background and Aims Febrile seizures are a common health problem in pediatric practice, knowledge of clinical manifestations and their evolution is important for correct therapeutic approach.

Assessment of clinical and evolutional features of febrile seizures (FS), with emphasis on risk factors such as age of onset, sex, FS category, familial history, etc., on the appearance of FS and on the risk of their recurrence is the aim of this work.

Method The authors conducted a retrospective study including 127 children aged 6 months to 5 years, hospitalized for FS between January 2008-March 2009 in our clinic. The role of risk factors in the development and recurrences of FS was analyzed.

Results FS appearance correlates with high fever (78\%), male (54\%), age $1-2$ years (50\%), but most do not associate familial history $(20 \%)$, the global presence of at least one risk factor is found in $93 \%$ of cases.

Recurrences appear in $43 \%$ of cases of FS and $98 \%$ of cases correlate with the presence of at least one risk factor for recurrences (familial history 16\% cases, onset of FS under the age of 1 year $29 \%$ cases, $47 \%$ complex FS).

Conclusions FS appearance correlates with high fever, male gender and age 1-2 years; appearance of relapses associates with complex FS, familial history and age under 1 year at onset.

\section{CLINICAL SPECTRUM OF CEREBRAL PALSY IN SOUTH JORDAN; ANALYSIS OF 122 CASES}

doi:10.1136/archdischild-2012-302724.1519

0 Nafi. Pediatric Neurology, Mutah University, Karek, Jordan 